\title{
FORAGING FOR THE SELF:
} ENVIRONMENT SELECTION FOR AGENCY INFERENCE

Kelsey Perrykkad ${ }^{1}$, Jonathan E. Robinson ${ }^{1}$ and Jakob Hohwy ${ }^{1}$

1. Cognition and Philosophy Lab, Philosophy Department, School of Philosophical, Historical and International Studies, Monash University 


\section{AbSTRACT}

Sometimes agents choose to occupy environments that are neither traditionally rewarding or worth exploring, but which rather promise to help minimise uncertainty related to what they can control. Selecting environments that afford inferences about agency seems a foundational aspect of environment selection dynamics - if an agent can't form reliable beliefs about what they can and can't control, then they can't act efficiently to achieve rewards. This relatively neglected aspect of environment selection is important to study so that we can better understand why agents occupy certain environments over others; something may also be relevant for mental and developmental conditions, such as autism. This online experiment investigates the impact of uncertainty about agency on the way participants choose to freely move between two environments, one that has greater irreducible variability and one that is more complex to model. We hypothesise that increasingly erroneous predictions about the expected outcome of agency-exploring actions can be a driver of switching environments; and we explore which type of environment agents prefer. Results show that participants actively switch between the two environments following increases in prediction error, and that the tolerance for prediction error before switching is modulated by individuals' autism traits. Further, we find that participants more frequently occupy the variable environment, which is predicted by greater accuracy and higher confidence than the complex environment. This demonstrates how participants forage to reduce uncertainty of action-outcome contingencies and support agency inferences.

Keywords: environmental niche selection, environment complexity, agency, autism, prediction error 
It is well understood that agents take an active part in choosing their environment to maximise information gain or to fulfill their needs and desires; that is, to achieve epistemic and pragmatic goals. However, in some cases, we pick an environment not for the reward it offers or even the information about it we can gain, but because it supports agent-focused epistemic foraging; we pick environments allowing us to manage uncertainty about action-outcome contingencies. To illustrate, consider someone turning down an exciting, well-paid promotion, not for fear of the responsibility that comes with increased decision-making powers, but because they are not confident they will be able to discern the power structures closer to the top of the management hierarchy; they know they will be in control, but the mapping of actions to outcomes threatens to be ambiguous - so they stay put. These decisions can seem perplexing to outsiders but there has been little research on these types of drivers for environment selection. In this study, we therefore explore how participants pick environments to forage for agency information.

Primarily, we focus on how agents choose which environments to occupy, and when to switch between available environments. When exploration and foraging studies traditionally consider the effect of uncertainty on these decisions, they are focused on uncertainty in the availability, stability and distribution of pragmatic rewards across available environments (Constantino \& Daw, 2015; Hutchinson, Wilke, \& Todd, 2008; Kacelnik \& Bateson, 1996; Mehlhorn et al., 2015). There is growing acknowledgement that, under a broad conception of reward, resources driving such decisions can include information gain or uncertainty reduction in addition to more traditional pragmatic rewards (Cohen, McClure, \& Yu, 2007; Friston et al., 2015; Inglis, 2000; Inglis, Langton, Forkman, \& Lazarus, 2001; Pezzulo \& Friston, 2019). This provides a richer perspective on environment selection behaviour, where an animal might forego traditional rewards in order to forage a different patch for information. Decreasing certainty in the current 


\section{ENVIRONMENT SELECTION FOR AGENCY INFERENCE}

environment, and expected increased certainty in the next one, may therefore drive a shift between environments.

Existing research has not focused much on how beliefs about one's own agency contribute to environment decisions. Beliefs about agency consist in judgements about the degree to which one can effectively act to bring about expected sensory consequences in the current environment, and uncertainty about agency then relates to factors that undermine the agent's ability to infer what they can efficiently control. There will be relatively little uncertainty about agency when foraging in an environment where all areas are easily accessible, and rewards are easy to collect when encountered. Uncertainty mounts when the mapping from actions to outcomes becomes ambiguous due to variability in the control states of the agent, such as cramps in their limbs or defects of their tools, or inability to ascertain the causal impact of their actions in the environment. The upshot is that increasing uncertainty about agency impedes successful action for either epistemic or pragmatic gain. While successful agency is in itself rewarding (Inglis, 2000), this approach contrasts with research focusing on uncertainty in choosing patches for foraging due to variability or volatility in the availability and depletion of extrinsic rewards.

The relationship between judgements of agency and ongoing environment selection is significant because it may throw new light on otherwise perplexing foraging decisions, where agents forego both pragmatic and informational rewards. This may speak to maladaptive symptoms of some mental conditions (Addicott, Pearson, Sweitzer, Barack, \& Platt, 2017), which often relate to action-outcome contingencies and inferred agency. Examples include learned helplessness as a model for depression (Vollmayr \& Gass, 2013), delusions of control or negative symptoms such as avolition in schizophrenia (Bentall et al., 2010; Jeannerod, 2008; Synofzik \& 
Voss, 2010), incorrect source attribution and intense responses to arbitrary sensory cues in posttraumatic stress disorder (Linson, Parr, \& Friston, 2020), and repetitive behaviours in autism spectrum condition (autism) (Perrykkad \& Hohwy, 2020a). In other words, it may be that agents occasionally choose environments that promote unambiguous agency beliefs, over more rewarding or informative environments. This can in many cases be rational since highly precise agency beliefs afford efficient pursuit of other rewards.

Here, we ask whether agents needing to judge their own agency under uncertainty will favour certain environments over others (environment selection), and what may motivate agents to abort foraging for agency information in one environment and shift to another (environment switching). To answer these questions specifically for agency beliefs, we distinguish our task from standard foraging tasks, such that there is no decreasing rate of resources in our environments. Instead we measure the incidental accumulation of uncertainty about agency, conceived as prediction error in the control of latent causes. In measuring a behavioural proxy for this prediction error (bPE), we hypothesise that agents will switch environments when this kind of prediction error increases (Cohen et al., 2007, p. 937; Mirza, Adams, Mathys, \& Friston, 2018). We also hypothesise that agents will on average spend more time foraging in environments that afford more accurate agency beliefs.

The environments presented to participants in this experiment are balanced in terms of how much bPE will accumulate but differ in underlying statistics and causal structure. One environment ("sand") is characterised by a relatively high level of irreducible variability in the opportunity for control, the other environment ("water") has less irreducible variability but instead is more causally complex. We aim to determine which type of environment best facilitates accurate 
judgements of agency, and which agents prefer. Variability and complexity are two factors that impact on environment decisions in general, but we did not have a hypothesis for how participants would weigh variability against complexity in environments to support judgements about their own agency.

As mentioned, environment selection dynamics may be particularly relevant for understanding behaviour in particular psychiatric conditions (Addicott et al., 2017). To begin exploring this, we consider autism traits, predicting that participants higher in autism traits will prefer to avoid the "water" environment, which calls for more complex, hierarchically deep internal models (Constant, Bervoets, Hens, \& Cruys, 2020; Perrykkad \& Hohwy, 2020b). Assuming autism traits are associated with models that struggle with greater uncertainty, we predict that participants with more autism traits will tolerate less bPE before switching environments (Perrykkad, Lawson, Jamadar, \& Hohwy, 2021).

Answers to these questions about foraging for agency-related information can ultimately help us better understand what drives agents' sometimes perplexing decisions about which environments to occupy and when to change environments.

\section{MethodS}

This study was approved by Monash University Human Research Ethics Committee (Project Number 26240) and was conducted in accordance with the relevant guidelines and regulations. All participants agreed to informed consent documents upon commencing the protocol 


\section{PARTICIPANTS}

A total of 229 participants were paid for completion of the study, 129 were recruited from Amazon Mechanical Turk using the Cloud Research platform (formerly TurkPrime (Litman, Robinson, \& Abberbock, 2017)), and 100 were recruited from Prolific (http://www.prolific.co ("Prolific," 2019)). Participants were paid \$4.50 USD (Amazon Mechanical Turk) or £4.10 GBP (Prolific) for completing the task, which took a median of 52 minutes to complete (including consent process and self-timed breaks, range: 26-146min total duration). Eligibility criteria included being fluent in English, aged 18-50, with no history of head injuries or neurological damage, normal or corrected-to-normal vision and no regular use of prescribed or unprescribed medication that may affect cognitive functioning.

We opted to use PsychoJS for stimulus presentation owing to its low latency and high precision timing (Anwyl-Irvine, Dalmaijer, Hodges, \& Evershed, 2020; Bridges, Pitiot, MacAskill, \& Peirce, 2020). Despite this, a pilot sample demonstrated that, due to the computational demand of the task, significant frame delays were present for many participants. For this reason, we chose stringent exclusion criteria to ensure relatively stable presentation in the final dataset. In the final dataset, a total of 148 participants were excluded for one or more of the following reasons: incomplete dataset recorded due to technical difficulties $(n=14)$, disqualification due to report of drug abuse in demographics survey $(n=1)$, average accuracy less than $25 \%$ (chance $=20 \%, \mathrm{n}=53$ ) or if more than $50 \%$ trials were defined as bad $(n=127$; see criteria below). Of the final dataset, nine participants reported diagnosed mental conditions (ADHD: $n=2$, Anxiety: $n=3$, Depression: n=2, PTSD: $n=1$, Dyslexia: $n=1$ ) but were not excluded from the study. 41 participants out of the final dataset of 84 (demographics in Table 1) were from Amazon Mechanical Turk, and 43 from Prolific. 
Table 1 - General Demographic Information

\begin{tabular}{|c|c|c|c|}
\hline Demographic & Category & $\mathbf{N}$ & $\begin{array}{r}\% \\
(n=84) \\
\end{array}$ \\
\hline \multirow[t]{3}{*}{ Gender } & Male & 59 & $70.2 \%$ \\
\hline & Female & 24 & $28.6 \%$ \\
\hline & Other & 1 & $1.2 \%$ \\
\hline \multirow[t]{5}{*}{ Age } & $18-24$ & 34 & $40.5 \%$ \\
\hline & $25-31$ & 20 & $23.8 \%$ \\
\hline & $32-38$ & 22 & $26.2 \%$ \\
\hline & $39-45$ & 6 & $7.1 \%$ \\
\hline & $46-50$ & 2 & $2.4 \%$ \\
\hline \multirow{14}{*}{$\begin{array}{l}\text { Country of } \\
\text { Residence }\end{array}$} & USA & 41 & $48.8 \%$ \\
\hline & Poland & 16 & $19.0 \%$ \\
\hline & United Kingdom & 6 & $7.1 \%$ \\
\hline & Portugal & 6 & $7.1 \%$ \\
\hline & Spain & 3 & $3.6 \%$ \\
\hline & Canada & 2 & $2.4 \%$ \\
\hline & France & 2 & $2.4 \%$ \\
\hline & Israel & 2 & $2.4 \%$ \\
\hline & Greece & 1 & $1.2 \%$ \\
\hline & Hungary & 1 & $1.2 \%$ \\
\hline & Czech Republic & 1 & $1.2 \%$ \\
\hline & Austria & 1 & $1.2 \%$ \\
\hline & Estonia & 1 & $1.2 \%$ \\
\hline & Netherlands & 1 & $1.2 \%$ \\
\hline \multirow{2}{*}{$\begin{array}{l}\text { First } \\
\text { Language }\end{array}$} & English & 53 & $63.1 \%$ \\
\hline & Other - Fluent in English & 31 & $36.9 \%$ \\
\hline \multirow{4}{*}{$\begin{array}{l}\text { Highest } \\
\text { Completed } \\
\text { Education }\end{array}$} & Less than Highschool & 2 & $2.4 \%$ \\
\hline & $\begin{array}{l}\text { Highschool or equivalent } \\
\text { including Vocational Training }\end{array}$ & 29 & $34.5 \%$ \\
\hline & $\begin{array}{l}\text { Bachelors, Honours, Associate, } \\
\text { or Professional Degree }\end{array}$ & 32 & $38.1 \%$ \\
\hline & Masters or Doctorate & 21 & $25.0 \%$ \\
\hline \multirow{3}{*}{$\begin{array}{l}\text { Employment } \\
\text { Status }\end{array}$} & Unemployed or Not Working & 9 & $10.7 \%$ \\
\hline & Student or Intern & 23 & $27.4 \%$ \\
\hline & Employed & 52 & $61.9 \%$ \\
\hline
\end{tabular}




\section{PROCEDURE}

Following the informed consent procedure, participants completed a general demographics survey, followed by the Autism-Spectrum Quotient (AQ) (Baron-Cohen, Wheelwright, Skinner, Martin, \& Clubley, 2001) and Self Concept Clarity Scale (SCCS) (Campbell et al., 1996). Then they were forwarded to Pavlovia (http://pavlovia.org) to complete the agency task. Finally, they completed the Subthreshold Autism Trait Questionnaire (SATQ) (Kanne, Wang, \& Christ, 2012) and were compensated via a completion code or link. To ensure consistency in participant experience, participants were asked to complete the task using a chrome or firefox browser, using a laptop or desktop and with an external mouse (not laptop trackpad or touchscreen).

\section{THE BEACH TASK: AgenCy TASK DESIGN}

This experiment was a variant of the Squares Task (Grainger, Williams, \& Lind, 2014; Perrykkad et al., 2021; Russell \& Hill, 2001; Williams \& Happé, 2009), the most commonly used judgement of agency task used in autistic populations (Perrykkad \& Hohwy, 2020b). Stimuli were presented online using PsychoJS (v2020.2) (Peirce et al., 2019). In this version of the task, there were four numbered squares on the screen in each trial (see Figure 1). The squares were randomly coloured with perceived-luminance matched shades of blue, red, purple and yellow on a grey background. Participants pressed and held a number key to select the relevant square. Square selection was visually represented to the participant by colouring border of the screen (an area that squares couldn't enter) with the selected square's colour. If a square was selected, all the squares moved when the mouse was moved and all the squares stopped with a tiny amount of jitter (to ensure participants didn't think it had frozen) when the mouse stopped, so participants had to both select a square and move in order to accurately complete the task. The selected square moved at half the speed of the other squares. This meant that participants could only gain high precision 
information on the selected square's present state but could also gather less precise information from the other stimuli. This square selection function allowed us to capture moment to moment hypothesis without using eye-tracking (cf. Perrykkad et al. (2021)), and as in foveal pursuit, the selected square would result in the most precise information, though more noisy information was available from other options. All squares moved faster than the actual mouse distance, to allow more screen wraps before the edge is hit, since repositioning the mouse using PsychoJS was not possible. To give participants more freedom of movement when this happens, they could also press

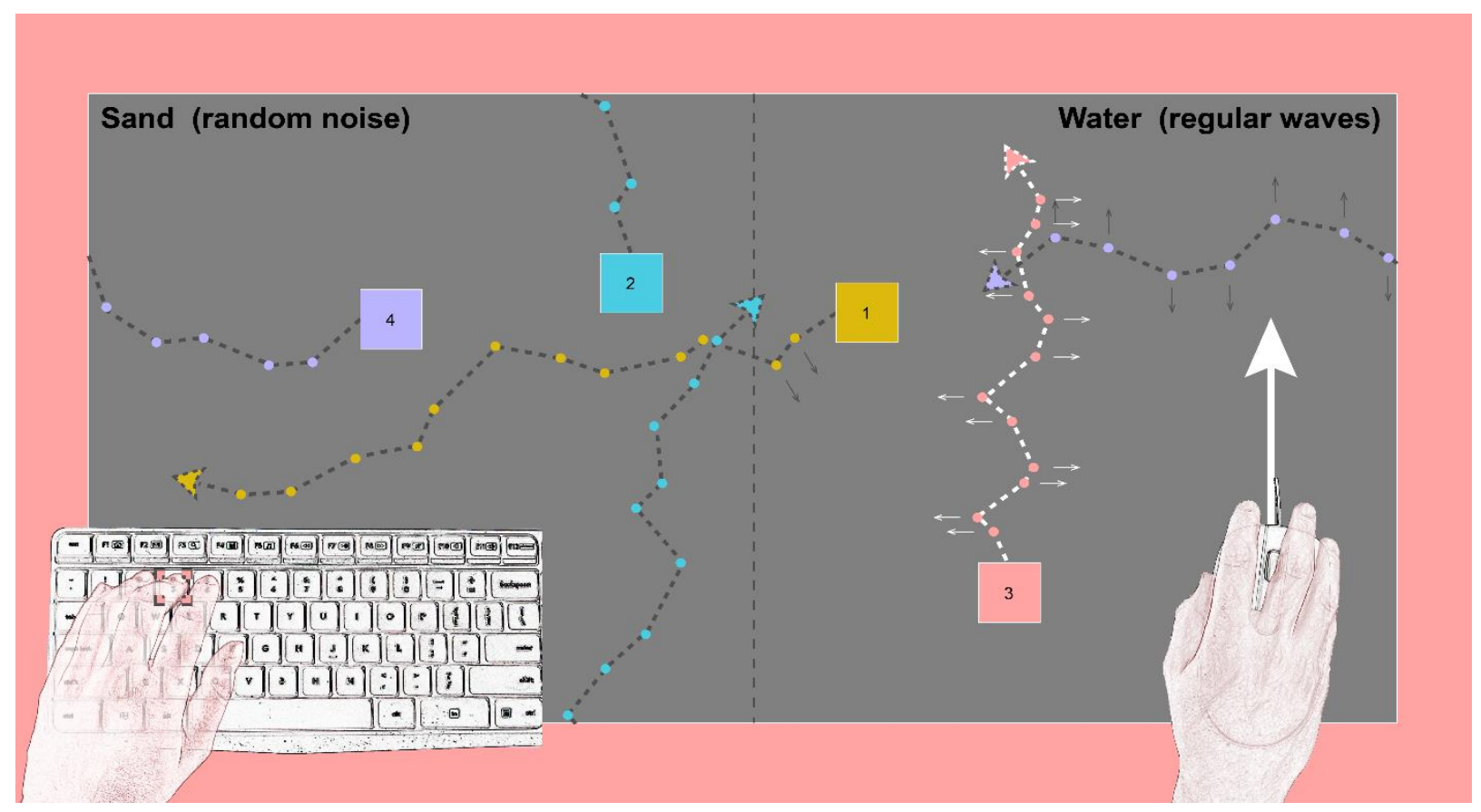

Figure 1 - Illustration of the Beach task

In this illustration, the participant has selected square 3 using their middle finger on the keyboard, which is indicated by the coloured border on screen. The mouse is being moved in a straight line forward, and the correct square (square 3) moves on average in this direction. Participants' task is to identify this square within 15s. Variability in direction is added to all squares and is sampled from the same distribution. The other squares move at an angle offset from the mouse position, e.g. square four moves $-90^{\circ}$ from the mouse position. The non-selected squares move faster. In this diagram, each dot represents a square position over a short period of time, and regular waves are depicted on the right half of the screen with small arrows indicating the forced direction at that time. Waves apply in the same direction for all squares in that environment on that frame, relative to the heading of the square. Had they been selected, squares 1 and 4 would count as having switched environment during the depicted period, while squares 2 and 3 have not. Please note that relative distances and wave duration depicted here are illustrative. Participants could only see the moving squares, grey background and selected coloured border. The centre boundary and environment labels were not visible. 


\section{ENVIRONMENT SELECTION FOR AGENCY INFERENCE}

' $Q$ ' to reposition all squares on the left half of the screen or ' $R$ ' to reposition all to the right. All square positioning (initial and after these button presses) was random.

Square positions were only updated at $30 \mathrm{hz}$, to limit computational performance variability across physical set ups. In what follows, we refer to a frame as one of these $30 \mathrm{hz}$ stimulus presentation data points. Regardless, refresh rate on monitors across participants was recorded as 60hz. Participants were given $15 \mathrm{sec}$ to identify the target square which they controlled.

Within each trial, there were two hidden environments. Half the screen (left or right) was randomly assigned water, and the other half sand. In both environments on each frame, for each square, a sample was taken from a $95 \%$ confidence interval for $\pm 70^{\circ}$. To save online processing costs, four random iterations of trial order and variability sampling for each square on each frame across the whole experiment were pre-established, and randomly selected for each participant. For squares in the sand environment, the variability angle was added to the input mouse angle from the participant (and the offset angle if the square is a distracter, described below) to generate the new square location for that frame (the distance moved depended on whether the square was currently selected). For squares located in the water environment, the sign of the sampled angle was forced to alternate between positive and negative values every 15 frames, creating regular waves, that pushed the participant consistently to the left or right of their heading. Since distribution and sampled magnitude of the variability was the same regardless of the environment, the displacement of the mouse on each frame across the two environments was equal. However, in water, there was an underlying predictable structure to the variability that was not present in sand. 
While the correct, controlled square followed the mouse movements with the addition of this variability, distracter squares moved at a random angle offset from the vector of mouse movement. This angle was also independently and randomly changed (and smoothly transitioned) five times in each trial, meaning that each distracter square appeared to turn five times when the participant did not initiate a turn, breaking any illusion of control resulting from motor adaptation. Half of the trials were no-control trials in which all four squares were distracter squares. After the $15 \mathrm{sec}$, all squares froze and were numbered, and an unspeeded numerical response was prompted from participants indicating which square they controlled or ' 0 ' if they thought they controlled none of the squares. Participants also responded on a 9-point likert scale to two additional questions asking for ratings of confidence ('Not at all', 'Very confident') and sense of agency ('No agency' to 'Complete agency'). Participants completed a total of 48 non-practice trials in three blocks of eight agentive trials and eight no-control trials.

An interactive, self-timed block of instructions before these task blocks explained the mechanics of the task and the presence of the two environments and included six timed, full practice trials (three control, three no-control). This served the purposes of both explaining the task and familiarising the participant to the movements of the correct square. Participants were explicitly informed that they would experience some variability in the movement of the squares. With respect to the environments, they were initially given screens filled with one environment at a time and differently coloured. On these screens, they were instructed that in sand, they would experience random jittering in their movement, while in the water, they would be pushed from side to side. Environments were not coloured in the main task (or the full practice trials), which had a grey background with the coloured border indicating the selected square. The colours of the squares during the practice period were different from the main task. During the full practice trials, 
participants were given feedback about the accuracy of their agency judgements, however the main task had no feedback. Without breaks or computational delays, the judgement of agency task was expected to take $20-25 \mathrm{~min}$.

We included additional measures to mitigate potential inattention in our online sample, including large warnings when participants failed to select squares or move the mouse during a trial, forced full screen at the start of every trial and instructional manipulation check questions during surveys (Oppenheimer, Meyvis, \& Davidenko, 2009).

\section{ANALYSIS}

Trials were defined as 'bad' and removed from further analysis if less than $50 \%$ of the frames were presented in the $15 \mathrm{~s}$ ( $<225$ frames), the average frame rate during non-lagged periods was less than half the rate expected, participants never pressed a square selection button, or participants moved the squares for less than $1 \%$ of the trial.

\section{Statistical AnAlysis}

All statistical analyses were conducted using Jamovi version 1.6.23 and the GAMLj module (Gallucci, 2019; R Core Team, 2018; The Jamovi Project, 2019). Many of the analyses described below are in the form of mixed linear models. Compared to traditional methods, this approach affords more sophisticated handling of missing and outlying data, thus improving the accuracy, precision, and generalisability of fixed effect estimates (Singmann \& Kellen, 2020). For each model, the structure will be specifically described below. Common control covariates for our analyses include number of frames and the standard deviation of wave duration in the water environment (participant averages were used where participant-wise data is used) to account for variability in stimuli presentation quality. Descriptive statistics of data quality and removal are 
outlined in the supplementary materials (Section 1). In addition, the survey measures are included as continuous fixed effects along with their two-way interactions with other fixed effects in each mixed model below. Since including both SATQ and AQ measures of autism traits in the mixed models would overlap greatly in variance accounted for, we decided to choose only the autism traits measure that was most orthogonal (least correlated) with our other survey measure (SCCS), and report the outcome at the start of the results section. For ease of interpretation, post-hoc tests for interactions with survey measures were simple effects contrasting participants mean scores to those above and below one standard deviation from the mean.

Across all statistical analyses, post-hocs are reported with a Bonferroni correction. For mixed models, the Satterthwaite method for estimating degrees of freedom is used. In order to validate the changes made due to practical considerations of putting the squares task online, we report supplementary results (Section 2) which validate the current methods by comparing results from Perrykkad et al. (2021) with the experiment reported here. The primary observed differences in behaviour are a more staccato movement style, driven by the button press function to change selected square. This leads to speed-related artefacts in bPE patterns, which are largely removed in the temporally sensitive analyses below by including speed in each relevant time window as a covariate fixed factor.

\section{ACCURACY}

Since there were equal numbers of trials where the ground truth of agency was present or absent, we computed signal detection theory measures for the agency signal for each participant. These included d' and criterion. Larger values of d' indicate greater sensitivity to the presence of a signal and would indicate greater unbiased accuracy on the judgement of agency task. A large 


\section{ENVIRONMENT SELECTION FOR AGENCY INFERENCE}

positive criterion value implies that the participant requires strong evidence before reporting that they had agency in a trial, while a smaller, negative criterion indicates that the participant is quite liberal with asserting agency. These values were tested using one-sample t-tests against a value of zero, indicating no sensitivity (d') or no bias (criterion) as the null hypothesis.

\section{ENVIRONMENTAL NICHE SELECTION}

Environment Bias. Trials were classified according to the dominant environment based on the percent of time spent in each sand and water in each trial. So, in a sand dominant trial, participants spent more than $50 \%$ of the trial in the sand half of the screen. To quantify an overall preference for one environment over the other, we computed two measures in each trial environment dominance (binary per trial) and percent of time spent in each environment (continuous). To test for overall environment preferences, one sample t-tests comparing these to $50 \%$ were used. To look at differences in accuracy or bias towards agency judgements depending on environmental preferences, signal detection theory measures split by environmental dominance were compared using paired sample t-tests for each d' and criterion.

Environment Switching. We were also interested in the way participants used switching between the available environments as a strategy to minimise prediction error and infer agency. Following Perrykkad et al. (2021), bPE was calculated by taking the Euclidean distance between where the selected square would have ended up if it had followed the trajectory of the mouse input (with a constant speed multiplier as described above) and where the square actually went. This means that bPE is largely under the control of the participants, and is influenced by three factors: 1) the speed of movement (determining the distance travelled, longer distances mean more bPE), 
and the angular changes to the input determined by 2) the uncertainty in the environment and 3) the angular offset if the selected square is not the correct one.

This allows us to investigate event related behavioural prediction error (by constructing an ERbPE, see Perrykkad et al. (2021)), around the time points when participants switched environments. Since environment switches that occurred concurrently with hypothesis switches could not be conceptually distinguished as a pure 'environment switch' strategy rather than primarily a hypothesis switch strategy, these switches were removed from these analyses to isolate the possible use of environment switching as an independent policy. As such, environment switch events here were due either to traversing the center or outer edges of the screen or pressing ' $\mathrm{Q}$ ' or ' $\mathrm{R}$ ' to switch to the other side of the screen.

In order to first select relevant datapoints for use in further analysis of bPE dataseries across relevant dimensions, we compare bPE around actual environment switches with a control dataseries. To test whether environment switching shows a distinct dataseries of bPE from traversing other regions of the screen, we generate a control time series locked to crossings of random positions on either side of the display. See supplementary materials for further details (Section 3). Average bPE for each frame in an epoch of \pm 15 frames (approximating $500 \mathrm{~ms}$ at $30 \mathrm{hz}$ presentation) were taken to generate a true (around true boundary crossing time points) and control (around eligible faux boundary crossings) ERbPE for each participant. Due to the sporadic movement observed in the experiment, the ERbPEs were split into lead-in and lead-out epochs which were defined by moving the ERbPE epoch trigger, if event is immediately preceded or followed by periods where nothing is selected, to the first (lead-out) or last (lead-in) time a hypothesis was validly selected. Difference wave sign-flip permutation testing $(10,000$ 
permutations) was used to determine frames in which the environment switch ERbPE differed from the control dataseries, and rankings of the true t-values against the maximum t-values from each permuted sample to provide estimated p-values for this analysis (non-parametric maximum pseudo t-test) that implicitly corrects for multiple comparisons.

To investigate the factors that contribute to differences in the ERbPE for true environment switches, a mixed model was used to compare bPE in the time window selected as significant by the permutation analysis. This model included the fixed factors of time bin and switch direction (to water or to sand). Given that movements were much more disjointed and speed was more variable (in ways we are not interested in) in the online version of the task, average speed in each frame for each participant was included as a covariate control fixed factor. By accounting for speed, this analysis focuses on displacement of the stimuli that is based in only the quality of hypothesis and environmental variability (interested readers are invited to see supplementary materials for a version of the model that does not include speed (Section 4), and one that replaces the dependent variable with speed (Section 5)). The standard fixed effects of SCCS and autism traits; standard control fixed effects of number of frames and wave time variability; and standard random effect of participant were also included. Only two-way interactions between autism traits, SCCS and other non-control fixed effects were included in this model.

Environment Selection. We then repeated two mixed models to look at factors within and following a trial that predicted the percent of time spent in each environment in each trial. The mixed model looking at how end of trial responses and trial type predict percent of time spent in each environment included fixed effects of sense of agency ratings, confidence, accuracy, judged agency and trial type. The standard fixed effects of SCCS and autism traits; standard control fixed 
effects of number of frames and wave time variability; and standard random effect of participant were also included. Only two-way interactions between autism traits, SCCS and other non-control fixed effects were included in this model.

The mixed model considering the association between in trial behaviours and percent of time spent in each environment included fixed effects of percent of frames spent moving, number of hypothesis switches, average bPE, average speed, acceleration and jerk. The standard fixed effects of SCCS and autism traits; standard control fixed effects of number of frames and wave time variability; and standard random effect of participant were also included. Only two-way interactions between autism traits, SCCS and other non-control fixed effects were included in this model.

\section{RESULTS}

In the present study we investigated environmental niche selection and switching using a judgement of agency task allowing participants to freely move a set of squares through two environments to determine which one they could reliably control. In addition, we collected measurements of confidence and sense of agency as well as measures of autism traits and selfconcept clarity.

\section{SuRVEYS}

Descriptive statistics for the survey measures are available in Table 2. Based on Pearson's correlations between the survey measures, AQ was chosen for inclusion in further models, as it was less correlated with SCCS $(r=-0.12, p=0.27)$ than SATQ was $(r=-0.25, p=0.02)$. 
Table 2 - Survey Descriptive Statistics

\begin{tabular}{llll} 
& Mean & Standard Deviation & Range \\
\hline AQ & 20.55 & 6.84 & $8-37$ \\
\hline SATQ & 26.80 & 10.88 & $5-51$ \\
\hline SCC & 40.31 & 10.82 & $20-60$ \\
\hline
\end{tabular}

\section{ACCURACY}

The average accuracy on the task was $54.07 \%$ (std: 15.7 , range: $26.32-87.18$ ), with chance at $20 \%$ (four squares or no-control). The signal detection theory analysis showed an average d' of 0.55 (std: 0.89 ) and an average criterion of -0.46 (std: 0.41$)$. One sample t-tests showed that both $d^{\prime}\left(\mathrm{t}(83)=5.68, \mathrm{p}=2.0 \times 10^{-7}, \mathrm{~d}=0.62\right)$ and criterion $\left(\mathrm{t}(83)=-10.285, \mathrm{p}=1.8 \times 10^{-16}, \mathrm{~d}=-1.12\right)$ were significantly different from zero. This shows that participants were sensitive to agency and had a liberal bias for attributing agency.

\section{ENVIRONMENTAL NiCHE SELECTION}

\section{ENVIRONMENT BIAS}

Participants spent an average of $49.00 \%$ (std: 3.54 ) of each trial in the water environment, and a one sample t-test showed a small but significant bias towards the sand environment $(\mathrm{t}(83)=-$ 2.62, $\mathrm{p}=0.010, \mathrm{~d}=-0.29$ ). Additionally, $47.50 \%$ of trials were classified as water dominant, and a one sample t-test showed that this too was significantly different from $50 \%(\mathrm{t}(83)=-2.65, \mathrm{p}=0.010$, $\mathrm{d}=-0.29)$. Paired sample t-tests comparing d' and criterion for water dominant and sand dominant trials showed no effect of environmental dominance $\left(d^{\prime}: \mathrm{t}(83)=-1.55, \mathrm{p}=0.13, \mathrm{~d}=-0.17\right.$; criterion: $\mathrm{t}(83)=0.74, \mathrm{p}=0.46, \mathrm{~d}=0.08)$. 


\section{ENVIRONMENT SELECTION FOR AGENCY INFERENCE}

\section{ENVIRONMENT SWITCHING}

On average, participants switched environments 7.38 times per trial (std:6.11, range: 0-67).

Of these, an average of $87.88 \%$ (std: 18.18) were traverses of the center line or outer edges, $11.86 \%$ coincided with a hypothesis switch (std: 17.94, removed from subsequent analyses) and $0.26 \%$ were performed using the button press mechanism which moved all squares to a chosen side of the screen (std: 2.95).

The permutation analysis compared bPE around true environment switches to a control dataset created from faux boundaries to identify any time points that show a distinct pattern of bPE (see Figure 2). In this analysis, the time of the event (both lead-in and lead-out times), five

a)

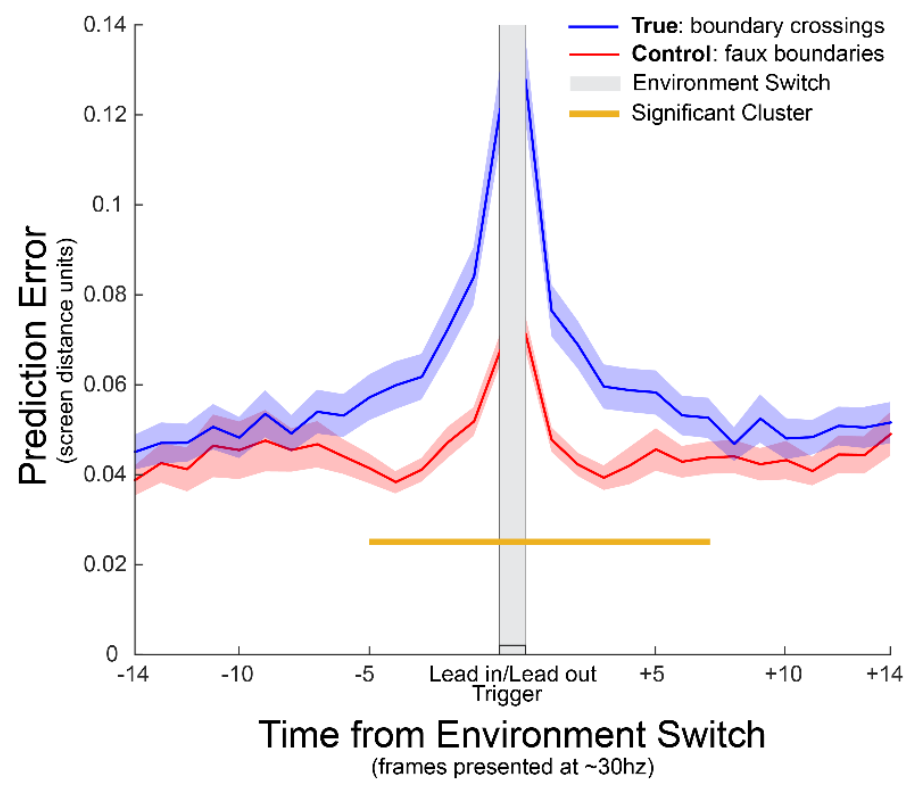

b)

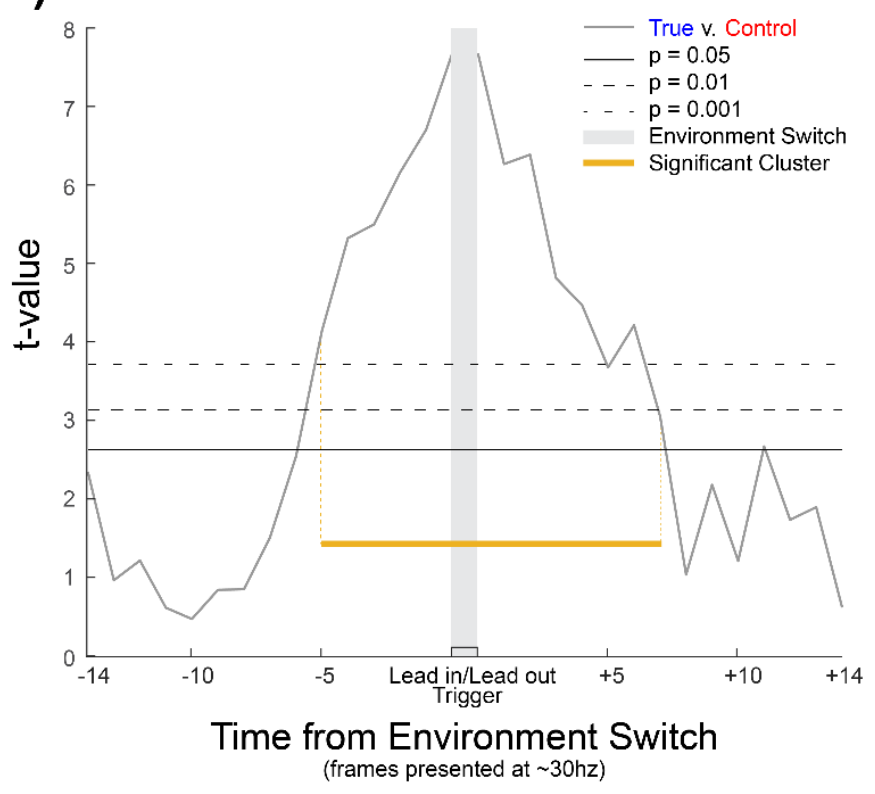

Figure 2 - Environment Switch ERbPE and Permutation Differences from Control Data

Panel a) Shaded regions represent standard error of the mean. The blue line (top) depicts behavioural prediction error (bPE) surrounding crossings of true environment boundaries time locked to the grey central period. The yellow bar indicates which of these timepoints is significantly different from a control dataset of bPE surrounding faux boundaries placed randomly between the true environmental boundaries (represented by the red line). Panel b) depicts the $t$-values at each time point resulting from permutation testing ranking the difference between the blue and red lines in panel a). Significance thresholds are represented by horizontal lines, and the cluster of time points which cross $\mathrm{p}=0.05$ are chosen for the yellow epoch represented in both panels, which is further analysed. 
datapoints preceding the lead-in time, and seven datapoints following the lead-out time were identified as significant which demonstrated these time points surpassed the $\alpha=0.05$ permuted $t$ threshold ( $\mathrm{t}_{\text {thresh }}=2.63$; see Figure $2 \mathrm{~b}$ ). Specifically this indicated that there were larger bPEs in this window around the environment switch than in other locations that participant traversed (mean difference $=0.0041$, std $=0.038$; see Figure 2a).

A mixed model was used to determine which factors influence the pattern of bPE in this window while controlling for speed of movement (Figure 3). Results showed a significant main effect of time bin $\left(F(13,2175)=51.16, p=6.052 \times 10^{-116}\right)$ and an interaction between AQ score and time bin $(\mathrm{F}(13,2168)=2.32, \mathrm{p}=0.0046)$. Post-hoc analysis revealed that while the time of the event was not different between lead in or lead out value $(\mathrm{t}(2173)=-0.83, \mathrm{p}=1.0)$, both of these values were significantly greater than all other time bins in this window ( $\mathrm{t}$-values range: $\mathrm{t}(2189)=3.18 \times 10^{-}$ ${ }^{8}($ lead in vs. -1$): \mathrm{t}(2211)=7.23 \times 10^{-53}$ (lead out vs. +6$)$ ). Significant increases in bPE become

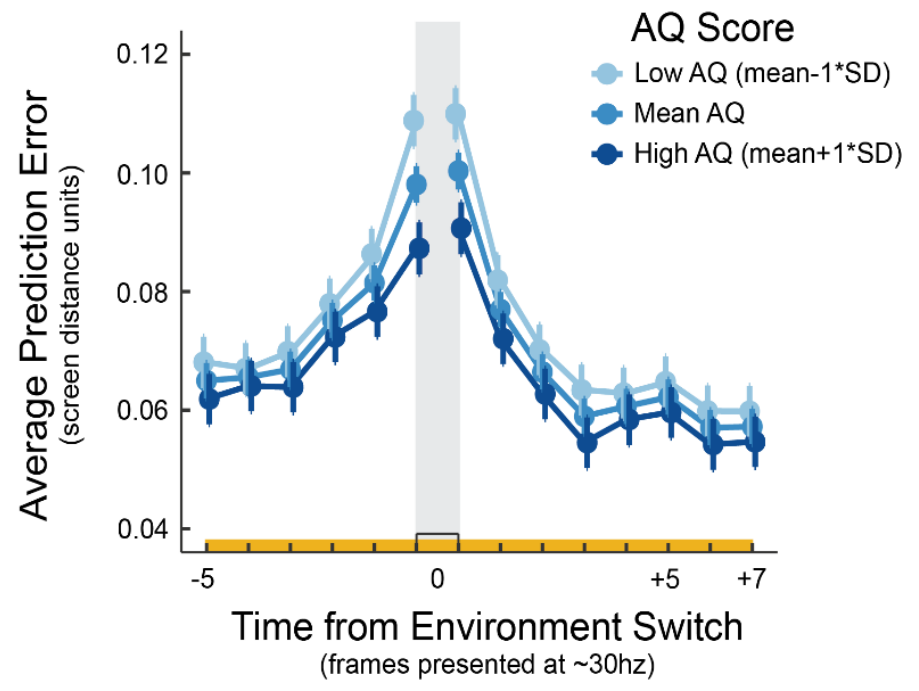

Figure 3 - Environment Switch ERbPE Split by AQ Score

Error bars represent standard error of the mean. Analysed time period matches the yellow bar in Figure 2. Behavioural prediction error here is adjusted for mean speed at each time. The low AQ group (scores of 13 or lower; $n=13$ ) are represented by the lightest blue, the high autism traits group (scored 28 or higher; $n=9$ ) are represented by the darkest blue, and the mean autism group scored between 14 and $27(n=62)$. 
apparent two frames preceding this peak, which, while not significantly greater than three frames preceding the switch $(\mathrm{t}(2175)=-3.27, \mathrm{p}=0.098)$, is significantly greater than four frames preceding $(\mathrm{t}(2174)=-3.80, \mathrm{p}=0.013)$. See supplementary materials for all comparisons (Section 6). bPE drops back to a stable value around two frames following the switch, which is statistically no different from three frames following $(\mathrm{t}(2174)=2.98, \mathrm{p}=0.26)$, but is still decreasing from the frame before $(t(2175)=4.20, p=0.0026)$. For the interaction, looking at simple effects of AQ by time bin showed that at both the lead in $\left(\mathrm{F}(1,169)=12.52, \mathrm{p}=5.21 \times 10^{-4}\right)$ and lead out $(\mathrm{F}(1,169)=10.04, \mathrm{p}=0.0018)$ times there was a significant effect of AQ, such that AQ score was inversely associated with bPE (see Figure 3).

\section{ENVIRONMENT SELECTION}

The mixed model used to investigate how time spent in each environment was predicted by responses at the end of the trial and trial type revealed main effects of confidence $(\mathrm{F}(1,2955)=7.58, \mathrm{p}=0.0059)$ and accuracy $(\mathrm{F}(1,2955)=4.44, \mathrm{p}=0.035)$. In correct trials, participants spent $1.79 \%$ longer in the sand environment. Further, as confidence increase, time spent in sand increases (mean diff of $2.70 \%$ between highest and lowest confidence trials).

The mixed model used to investigate how time spent in each environment was predicted by other behaviours during the trial had no significant main effects or interactions.

\section{DISCUSSION}

Unless we have security in our ability to manage uncertainty around action-outcome mappings, we cannot be confident we can act successfully. So, before we can act for epistemic or pragmatic gain, it is reasonable to favour an environment that affords agency-focused information 
foraging. In the experiment reported here, participants were able to freely move between two environments, a variable "sand" environment and a complex "water" environment as they tried to determine which of four squares on the screen they controlled, if any. The results show that participants have a slight preference for the sand environment, which was predicted by greater accuracy and confidence. Further, the accumulation of prediction error in the control of movements foreshadows environment switching, and participants with fewer autism traits tolerated more prediction error before they opted to abandon their current foraging context.

Of the two environments offered to participants in this experiment, there are a priori benefits of each. They were matched for prediction error at the most basic level - that is, the Euclidean distance between where the objects would have ended up had they followed the mouse and where they did end up in each environment was the same. This yields the basic measure of bPE in our results. However, in the sand environment, there was no further structure underlying the variability in the environment. Successfully modelling the variability at this most basic level is the most one can reduce their uncertainty in this environment. In the water environment, given a good model, one could predict which direction the variability would be confined to, essentially halving the range of expected locations, and reducing the surprise associated with large displacements provided they fall within the expected direction. In this sense, the two environments pitted model complexity (modelling both the waves and the variability in the water environment) against irreducible uncertainty (the full range of variability experienced in the sand environment).

Our results suggest that participants did have a significant albeit slight preference for the sand environment, preferring reduced model complexity to the reducible uncertainty afforded by the water environment, for the purposes of the agency-focused foraging task. This was a modest 
preference, with a mean difference of only one percent across trials, but it was reliable across different ways of measuring preference, with an average of $2.5 \%$ more trials being classified on the whole as sand dominant. This also did seem to garner a quantifiable advantage, with participants spending longer in the sand environment when they were correct, and participants reporting greater confidence with more time spent in the sand environment. Future work could titrate the variability to push participants towards greater model complexity or greater irreducible uncertainty over multiple trials to establish individual participant thresholds that may be associated with participant differences such as autism traits or self-concept clarity. While we expected that higher autism traits would predict stronger preference for environments that require lower model complexity, the preference for sand was not dependant on AQ score. Both environments were very simple, and future experiments should consider naturalistic environments with more hidden causes to uncover possible effects of AQ on environment preference. The validation of this task in the online setting makes these kinds of manipulations particularly accessible.

The pattern of prediction error (bPE) around environment switches suggests that participants were using a switch in environment (in either direction) as an intentional response to bPE, as demonstrated by the permutation testing results. The ERbPE mixed model showed that even when controlling for speed, the other factors contributing to bPE magnitude also increased before participants switched environments, and decreased afterward - namely, environmental variability and square offset (when the selected square was not the correct one). This is compelling evidence that participants are using the act of switching environments as a response to increasing bPE. 
The way environment switching is used as a strategy in response to bPE was modulated by participants' autism traits. Participants with high AQ appear to tolerate less prediction error before acting in response to it, a finding that is consistent with AQ modulation of the hypothesis switch ERbPE reported in Perrykkad et al. (2021). Since bPE does not account for improved prior expectations based on successful modelling of the environment, these results might also indicate that lower autistic traits scores are associated with better modelling of the environment, thus while having higher bPE at the time of switching environments, they cognitively experience similar quantities of prediction error to participants with higher autism traits when choosing to act. Future work using neural measures of prediction error may be able to tease this apart.

We note that the use of autism traits rather than a clinically diagnosed population places limits to the generalisability of this result. While overall the sample size in post-hoc analyses used to compare interactions with autism traits is low, the omnibus interactions were based on modelled trends in the full dataset of continuous AQ scores. Nevertheless, environmental uncertainty might be particularly relevant to agency-focused foraging for different levels of autistic traits and we do show interactions between environment selection and AQ. These are worth following up in future studies in diagnosed populations.

Methodologically, the results of this online study demonstrate the robustness of the squares task in answering fine-grained questions despite increased noise from button press mechanics, internet/computing instability and even without the use of eye-tracking for hypothesis tracking (cf. Perrykkad et al. (2021), see supplementary materials sections 1-2).

In the broader perspective, revealing how active foraging for agency-related information contributes to environment preferences can help us better understand agents' sometimes 
perplexing choices to forego environments where they could forage for traditional rewards or information about the world. This may also help understand the environments preferred in some mental and developmental conditions. In a more generic learning context, perhaps foraging for agency-related information occurs because it helps agents solve the credit assignment problem, familiar from reinforcement learning (Rothkopf \& Ballard, 2010). By pre-emptively ensuring they have a model of what they can and cannot precisely control in a given environment, agents will have a better fix on which parts of their actions should be credited with obtaining them their rewards. Overall, such findings complement the existing picture of factors at play (Mehlhorn et al., 2015) in foraging decisions including the rate and variability of resource depletion, and other factors, such as individual and social factors.

\section{Availability of Data AND Materials}

Analysis scripts developed for this project are freely available on Github (https://github.com/kperrykkad/beach-task/), and the dataset used for statistical analysis is freely available on Figshare (https://doi.org/10.26180/14826579). 


\section{REFERENCES}

Addicott, M. A., Pearson, J. M., Sweitzer, M. M., Barack, D. L., \& Platt, M. L. (2017). A primer on foraging and the explore/exploit trade-off for psychiatry research. Neuropsychopharmacology, 42(10), 1931-1939.

Anwyl-Irvine, A., Dalmaijer, E. S., Hodges, N., \& Evershed, J. K. (2020). Realistic precision and accuracy of online experiment platforms, web browsers, and devices. Behavior research methods. doi:10.3758/s13428-020-01501-5

Baron-Cohen, S., Wheelwright, S., Skinner, R., Martin, J., \& Clubley, E. (2001). The Autism-Spectrum Quotient (AQ): Evidence from Asperger Syndrome/High-Functioning Autism, Malesand Females, Scientists and Mathematicians. Journal of Autism and Developmental Disorders, 31(1), 5-17. doi:10.1023/A:1005653411471

Bentall, R. P., Simpson, P. W., Lee, D. A., Williams, S., Elves, S., Brabbins, C., \& Morrison, A. P. (2010). Motivation and avolition in schizophrenia patients: The role of self-efficacy. Psychosis, 2(1), 1222. doi:10.1080/17522430903505966

Bridges, D., Pitiot, A., MacAskill, M. R., \& Peirce, J. W. (2020). The timing mega-study: comparing a range of experiment generators, both lab-based and online. PeerJ, 8, e9414.

Campbell, J. D., Trapnell, P. D., Heine, S. J., Katz, I. M., Lavallee, L. F., \& Lehman, D. R. (1996). Selfconcept clarity: Measurement, personality correlates, and cultural boundaries. Journal of personality and social psychology, 70(1), 141.

Cohen, J. D., McClure, S. M., \& Yu, A. J. (2007). Should I stay or should I go? How the human brain manages the trade-off between exploitation and exploration. Philosophical Transactions of the Royal Society B: Biological Sciences, 362(1481), 933-942.

Constant, A., Bervoets, J., Hens, K., \& Cruys, S. V. d. (2020). Precise Worlds for Certain Minds: An ecological perspective on the relational self in autism. TOPOI, 39(3), 611-622. doi:10.1007/s11245-018-9546-4

Constantino, S. M., \& Daw, N. D. (2015). Learning the opportunity cost of time in a patch-foraging task. Cognitive, Affective, \& Behavioral Neuroscience, 15(4), 837-853. doi:10.3758/s13415-015-0350$\mathrm{y}$

Friston, K., Rigoli, F., Ognibene, D., Mathys, C., Fitzgerald, T., \& Pezzulo, G. (2015). Active inference and epistemic value. Cognitive neuroscience, 6(4), 187-214.

Grainger, C., Williams, D., \& Lind, S. E. (2014). Online Action Monitoring and Memory for SelfPerformed Actions in Autism Spectrum Disorder. Journal of Autism and Developmental Disorders, 44, 1193-1206.

Hutchinson, J. M., Wilke, A., \& Todd, P. M. (2008). Patch leaving in humans: can a generalist adapt its rules to dispersal of items across patches? Animal Behaviour, 75(4), 1331-1349.

Inglis, I. (2000). The central role of uncertainty reduction in determining behaviour. Behaviour, 137(12), 1567-1599.

Inglis, I., Langton, S., Forkman, B., \& Lazarus, J. (2001). An information primacy model of exploratory and foraging behaviour. Animal Behaviour, 62(3), 543-557. doi:https://doi.org/10.1006/anbe.2001.1780

Jeannerod, M. (2008). The sense of agency and its disturbances in schizophrenia: a reappraisal. Experimental Brain Research, 192(3), 527. doi:10.1007/s00221-008-1533-3

Kacelnik, A., \& Bateson, M. (1996). Risky Theories-The Effects of Variance on Foraging Decisions. American Zoologist, 36(4), 402-434. doi:10.1093/icb/36.4.402

Kanne, S. M., Wang, J., \& Christ, S. E. (2012). The Subthreshold Autism Trait Questionnaire (SATQ): development of a brief self-report measure of subthreshold autism traits. J Autism Dev Disord, 42(5), 769-780. doi:10.1007/s10803-011-1308-8 
Linson, A., Parr, T., \& Friston, K. J. (2020). Active inference, stressors, and psychological trauma: A neuroethological model of (mal) adaptive explore-exploit dynamics in ecological context. Behavioural Brain Research, 380, 112421.

Litman, L., Robinson, J., \& Abberbock, T. (2017). TurkPrime. com: A versatile crowdsourcing data acquisition platform for the behavioral sciences. Behavior research methods, 49(2), 433-442.

Mehlhorn, K., Newell, B. R., Todd, P. M., Lee, M. D., Morgan, K., Braithwaite, V. A., . . . Gonzalez, C. (2015). Unpacking the exploration-exploitation tradeoff: A synthesis of human and animal literatures. Decision, 2(3), 191-215. doi:10.1037/dec0000033

Mirza, M. B., Adams, R. A., Mathys, C., \& Friston, K. J. (2018). Human visual exploration reduces uncertainty about the sensed world. PloS one, 13(1), e0190429. doi:10.1371/journal.pone.0190429

Oppenheimer, D. M., Meyvis, T., \& Davidenko, N. (2009). Instructional manipulation checks: Detecting satisficing to increase statistical power. Journal of experimental social psychology, 45(4), 867-872.

Pavlovia. Retrieved from https://pavlovia.org/

Peirce, J., Gray, J. R., Simpson, S., MacAskill, M., Höchenberger, R., Sogo, H., . . Lindeløv, J. K. (2019). PsychoPy2: Experiments in behavior made easy. Behavior research methods, 51(1), 195-203.

Perrykkad, K., \& Hohwy, J. (2020a). Fidgeting as self-evidencing: A predictive processing account of nongoal-directed action. New Ideas in Psychology, 56, 100750. doi:https://doi.org/10.1016/j.newideapsych.2019.100750

Perrykkad, K., \& Hohwy, J. (2020b). Modelling Me, Modelling You: the Autistic Self. Review Journal of Autism and Developmental Disorders, 7, 1-31. doi:10.1007/s40489-019-00173-y

Perrykkad, K., Lawson, R. P., Jamadar, S., \& Hohwy, J. (2021). The effect of uncertainty on prediction error in the action perception loop. Cognition, 210, 104598. doi:https://doi.org/10.1016/j.cognition.2021.104598

Pezzulo, G., \& Friston, K. J. (2019). The value of uncertainty: An active inference perspective. Behavioral and Brain Sciences, 42. doi:http://dx.doi.org/10.1017/S0140525X18002066

Prolific. (2019). Retrieved from http://www.prolific.co

Rothkopf, C., \& Ballard, D. (2010). Credit Assignment in Multiple Goal Embodied Visuomotor Behavior. Frontiers in psychology, 1(173). doi:10.3389/fpsyg.2010.00173

Russell, J., \& Hill, E. L. (2001). Action-monitoring and Intention Reporting in Children with Autism. Journal of Child Psychology and Psychiatry, 42(3), 317-328. doi:10.1111/1469-7610.00725

Synofzik, M., \& Voss, M. (2010). Disturbances of the Sense of Agency in Schizophrenia. In B. Michela (Ed.), Neuropsychology of the Sense of Agency: From Consciousness to Action (pp. 145-155). Milano: Springer Milan.

Vollmayr, B., \& Gass, P. (2013). Learned helplessness: unique features and translational value of a cognitive depression model. Cell and Tissue Research, 354(1), 171-178. doi:10.1007/s00441-0131654-2

Williams, D., \& Happé, F. (2009). Pre-conceptual aspects of self-awareness in autism spectrum disorder: The case of action-monitoring. Journal of Autism and Developmental Disorders, 39(2), 251-259.

\section{ACKNOWLEDGEMENTS}

The authors appreciate the time and effort of their participants without whom this research would not be possible. This work is supported by the Australian Research Council (DP190101805)

to Jakob Hohwy. Kelsey Perrykkad was supported by the Australian Government Research Training Program. The authors declare no conflicts of interest. 